\title{
Development of telemedicine tools with an emphasis on visual observation
}

\author{
Masato Takahashi ${ }^{1} \cdot$ Reimei Koike $^{1} \cdot$ Kazuki Nagasawa $^{1} \cdot$ Yasuhiro Manabe $^{2} \cdot$ Hirofumi Hirana $^{2} \cdot$ \\ Mitsuyuki Takamura ${ }^{3} \cdot$ Tetsuya Hongawa $^{4} \cdot$ Izumi Kimoto $^{5} \cdot$ Keiko Ogawa-Ochiai $^{6} \cdot$ Norimichi Tsumura $^{7}$
}

Received: 9 September 2021 / Accepted: 27 December 2021 / Published online: 22 January 2022

(C) International Society of Artificial Life and Robotics (ISAROB) 2022

\begin{abstract}
We developed a system to improve the quality of telemedicine, and the test results obtained have been presented in this paper, along with the technical details of the system. The spread of COVID-19 has accelerated the need for telemedicine to effectively prevent infections. However, in traditional Japanese medicine (Kampo), where color is essential, an accurate diagnosis cannot be made without color reproduction. Because commercial smartphones cannot reproduce colors with the level of fidelity required for medical treatments, we created a color chart that includes the human skin and tongue colors to help doctors identify their colors accurately during a telemedicine examination. Further, we developed a telemedicine system that allows for automatic color correction using a mobile device, with a color chart and non-contact heart rate measurements.
\end{abstract}

Keywords Telemedicine $\cdot$ Color reproduction $\cdot$ Color-chart $\cdot$ Pulse rate $\cdot$ Remote photoplethysmograph

\section{Introduction}

The demand for telemedicine services is increasing, and their value in the global market is expected to expand from US\$ 2.68 billion in 2016 to US\$22.71 billion in 2025 [1].

Masato Takahashi and Reimei Koike contributed equally to this work.

Masato Takahashi

mat_diver@chiba-u.jp

1 Graduate School of Science and Engineering, Division of Creative Engineering, Chiba University, 1-33 Yayoi-cho, Inage-ku, Chiba 263-8522, Japan

2 Shinseikai Toyama Hospital, 89-10 Shimowaka, Imizu City, Toyama 939-0243, Japan

3 Mie University Hospital, 2-174 Edobashi, Tsu, Mie 514-0001, Japan

4 The Mutual Aid Association of Prefectural Government Personnel, Shizuoka Prefectural Government Office Clinic, 9-6, Otemachi, Aoi-ku, Shizuoka 420-8601, Japan

5 Hirose Clinic, 6-37, Wkamatsu-cho, Kariya-shi, Aichi 448-0858, Japan

6 Kampo Clinical Center, Department of General Medicine, Hiroshima University Hospital, 1-2-3 Kasumi Hiroshima-city, Hiroshima 734-8551, Japan

7 Graduate School of Engineering, Chiba University, 1-33 Yayoi-cho, Inage-ku, Chiba 263-8522, Japan
Telemedicine services include online medical care and doctor-to-doctor communication, specialist advice to doctors, home monitoring of chronic diseases, and physical therapy guidance. With the spread of COVID-19, infection control in healthcare is also an essential factor. To prevent the spread of infections, face-to-face encounters between patients in medical facilities and between clinicians and patients need to be alleviated. Telemedicine is used because it helps avoid face-to-face encounters [2-4]. Smartphones are used to monitor human health to detect biometric information such as heart rate, respiration rate, sleep patterns, and activity levels. However, a few of these health apps are reported to lack sufficient quality [5]. Meanwhile, smartphones are used for telemedicine in ophthalmology and otorhinolaryngology $[6,7]$.

The development of video communication systems has been remarkable, and they are being used for conferences, classes, and academic conferences. However, color management in videoconferencing systems emphasizes the reproduction of preferable colors, which leads to incorrect judgements regarding the color of a person's skin or tongue in telemedicine. The importance of color reproduction in medical care has already been highlighted [8].

In Japan, the spread of COVID-19 has led to a rapid increase in the need for telemedicine from the perspective of infection prevention, and the Japanese government has implemented measures to promote online medical care, 
such as revising medical fees and allowing utilization from the first visit [9]. The revised "Guidelines for the Appropriate Implementation of Online Medical Treatment" issued by the Ministry of Health, Labor and Welfare in Japan in July 2019 [10] also states that, “....before implementing online medical care, it is desirable to actually conduct tests using information and communication devices to confirm the color and operability of images obtained through the devices". However, no specific color management method has been proposed thus far. The following processes need to be managed for accurate color reproduction: image input, processing, transmission, and image output. The input section needs to consider the characteristics of the ambient light, lighting, and camera; the processing and transmission section needs to correct color and prevent degradation; and the output section needs to adjust for the luminous characteristics of the monitor and ambient light. Such color management techniques require specialized knowledge and corrective equipment to be performed accurately. Therefore, doctors who practice vision-oriented medicine have been demanding simple and accurate color reproduction systems. In particular, Kampo doctors need to observe the color of a patient's face and tongue.

Kampo doctors use all five senses in their practice. The five senses are categorized as follows: "inspection" by sight, "listening and smelling examination" by hearing and smell, "inquiry" by listening to the patient's condition and subjective symptoms, and "palpation" by touching the patient. In "inspection", the doctor observes the patient's movements, skin color, and tongue signs [11]. We have been developing a telemedicine system using color charts and RGB cameras to address the needs of Kampo doctors who emphasize visual observation [12].

This report describes the implementation of the system on ISO devices such as iPhone and iPad, and the verification results for practical application.

\section{Methods and results}

This section provides an overview of the developed telemedicine system, improvement of the color chart, evaluation of the automatic color correction module using the color chart, and evaluation of the pulse wave measurement module.

\subsection{Overview of Telemedicine Tools}

Figure 1 shows an overview of the developed telemedicine tool. The system has two main functions: color correction to correctly grasp the color of the face and tongue; and

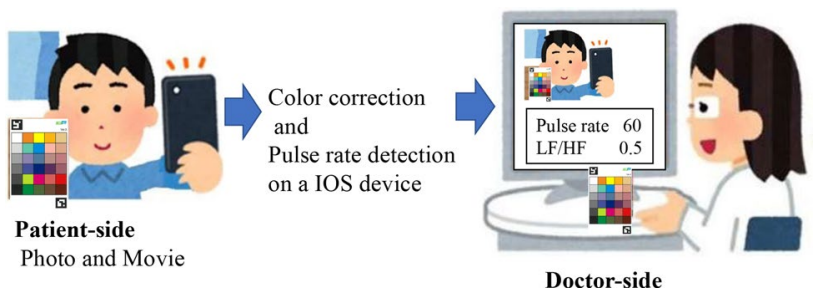

Fig. 1 Overview of telemedicine systems

(a) Screenshot of the initial menu screen

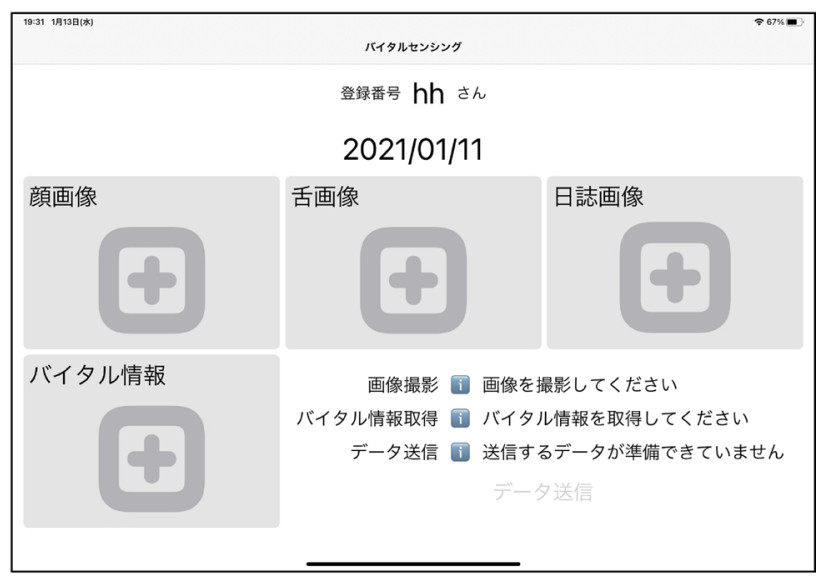

(b) Screenshot of all acquired data

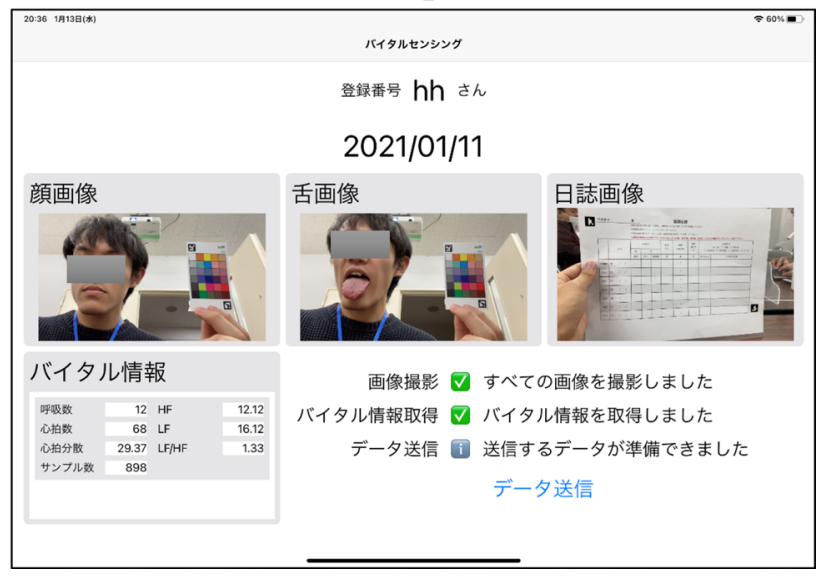

Fig. 2 Screenshot of the telemedicine system using an iPad on the patient's side

pulse wave measurement, which is necessary for auscultation. The system also helps import images of questionnaires written by patients. These functions were selected based on the requests of Kampo doctors. The color chart is used for color correction; the patient uses an iOS device such as an iPhone or iPad, and the doctor uses a personal computer. The color correction function was based on the method we proposed in a previous paper [12]. The pulse wave is obtained from the hemoglobin value, by signal processing the video captured by the RGB camera [13, 14]. 
Figure 2 shows the screen of the patient's iPad in Japanese. The initial screen shows the items to be executed by the patient. The upper left side of the screen is the face image; the center is the tongue image; and the right side is the logbook image. The lower left side of the screen shows the vital information obtained from the video. The patient performs four types of input operations. The patient executes the input operations in sequence, and the input information will be displayed. Vital information is shown as numerical values analyzed from the video. When the patient finishes all the input operations, the doctor will send the information via SNS. Currently, the information is sent via SNS called LINE. A series of operations are executed daily, and the data are accumulated sequentially.

Next, we explain the system on the doctor's side. Figure 3 shows the screen of the doctor's PC. The doctor can monitor the information sent from the patient through the SNS on the PC screen. The accumulated data can be used to observe the patients over time. The system is currently designed in Japanese; however, English is used in the figure for an explanation. When the doctor selects the list in the upper row on the PC screen, the patient's information is displayed. The bottom-right corner of the screen allows the doctor to add comments. The patient's image displayed on the screen is automatically color-corrected. Clicking on a patient's image increases the size of the image.

\subsection{Color chart improvements}

The improved color chart for Ver.3 is shown in Fig. 4. The color chart is modified from Ver.2, which was used in a previous study [12], and modified based on feedback from doctors to improve ease of use. The specifications are the same as in Ver.2, and the chart was printed on matte paper using an industrial inkjet printer. The improvements were only in the arrangement of color patches. The color-related data of the color charts are listed in Table 1.

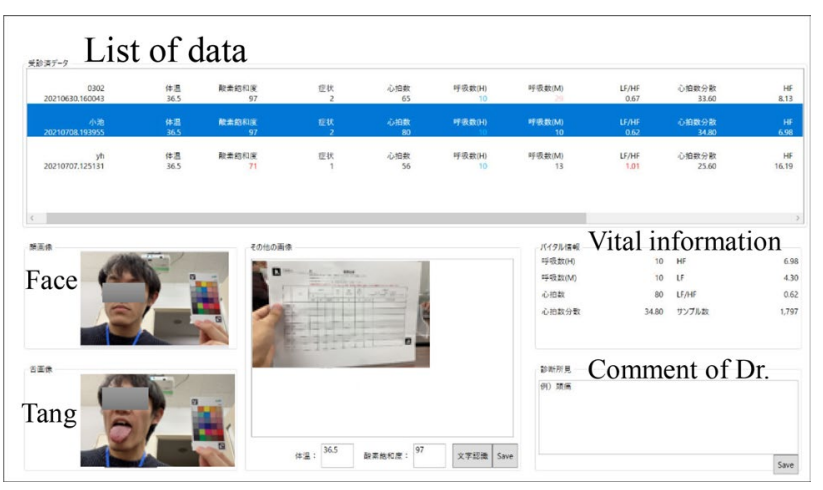

Fig. 3 Screenshot of the telemedicine system using a Windows PC on the doctor's side

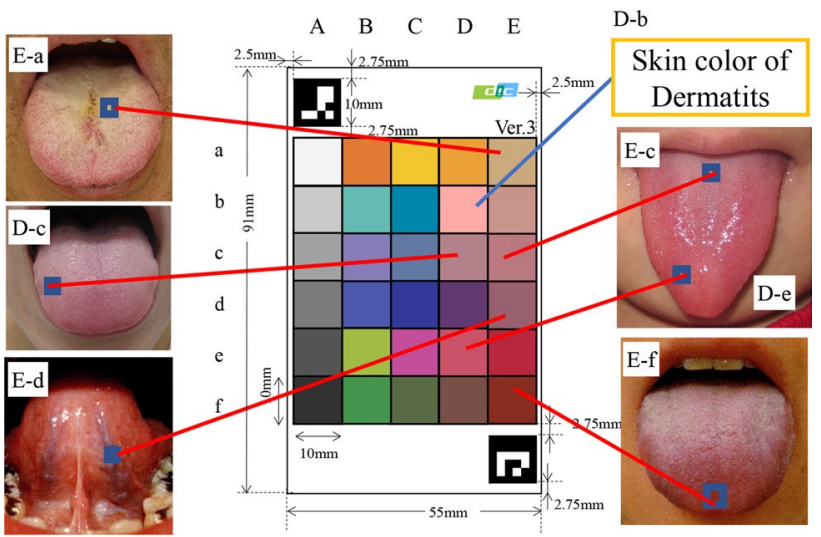

Fig. 4 Proposed color chart Ver.3 arrangement and selection of seven important colors

The color chart was improved through trial and error by consulting with Kampo doctors. We finally concluded that it would be better to arrange similar colors together, which led to the color chart Ver.3. In the revision process, we gathered similar colors, respecting the doctors' opinion that it would be easier to use if similar colors were placed side by side.

The color chart was established for use in the Asian race and needs to be improved for application to the global population. However the tongue color is of a mucous membrane, it can be adaptable to all humans.

\subsubsection{Evaluate the usability of the color chart}

To evaluate whether the color charts were easier to see, we interviewed three Chinese medicine doctors and presented them with Ver.2 and Ver.3.

Figure 5 shows the color chart presented for the evaluation. Three of the subjects used color chart Ver.2. The results showed that all three doctors agreed that the improved Ver.3 color chart was easier to read than the Ver.2 color chart. Two comments were included on the color chart, which have been listed in Table 2.

Table $1 L^{*} a^{*} b^{*}$ color values for the proposed color chart Ver.3

\begin{tabular}{llllll}
\hline & $\mathrm{A}$ & $\mathrm{B}$ & $\mathrm{C}$ & $\mathrm{D}$ & $\mathrm{E}$ \\
& $L^{*}, a^{*}, b^{*}$ & & & \\
\hline a & $97,0,0$ & $62,38,55$ & $82,6,74$ & $72,22,62$ & $72,8,22$ \\
b & $87,0,0$ & $70,-30,-4$ & $51,-21,-30$ & $78,30,15$ & $67,20,14$ \\
c & $76,0,0$ & $55,14,-30$ & $51,0,-25$ & $60,20,5$ & $58,27,7$ \\
d & $64,0,0$ & $40,14,-47$ & $28,24,-55$ & $30,27,-26$ & $48,25,2$ \\
e & $51,0,0$ & $73,-22,54$ & $50,54,-18$ & $52,50,13$ & $42,57,24$ \\
f & $36,0,0$ & $56,-37,30$ & $43,-12,18$ & $38,17,12$ & $33,40,30$
\end{tabular}

The $L^{*} a^{*} b^{*}$ color value is calculated by using white reference plate under D50 light source 


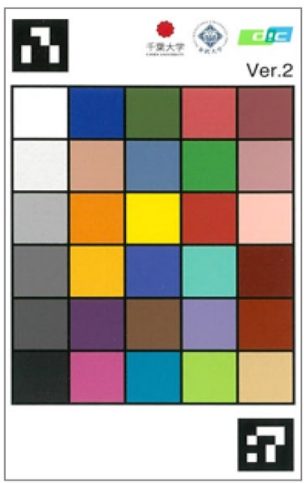

(a) Ver.2 Previous version

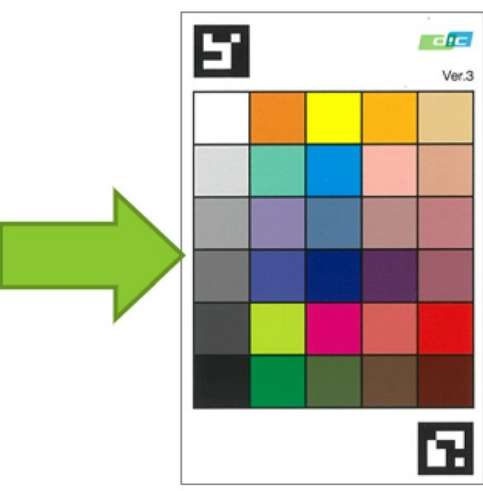

(b) Ver.3 Improved version

Fig. 5 Comparison of the color chart before and after the improvement

Table 2 Evaluation of the easier to see improved color chart

\begin{tabular}{l} 
No comments \\
\hline 1 The colors are close to actual \\
living organisms, making it \\
easier to compare and contrast \\
2 The patches are arranged in a \\
similar color gradient, which \\
makes it easier to compare \\
them
\end{tabular}

Step2 (on line) Camera image correction on The patient's side Adjust the RGB data of every color patches captured by the camera to match the reference RGB data. Adjust every color patches (ref. RGB data ) displayed on the monitor to the color patches on the color chart.
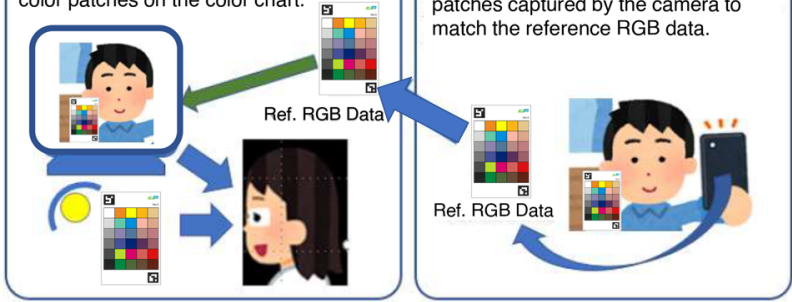

Fig. 6 Overview of automatic color correction method

\subsection{Accuracy evaluation of the automatic color correction module using the color chart}

\subsubsection{Automatic color correction using the color chart}

Automatic color correction using a color chart was performed in two steps, as shown in Fig. 6. By matching the colors of every patch on the color chart, the image taken will be displayed correctly on the doctor's monitor. The first step (offline) is to adjust every color patch (using RGB data) displayed on the monitor to match the color patch on the actual color chart. The second step (online) is to adjust the RGB data of every color patch captured by the camera to match the reference RGB data.

\subsubsection{Accuracy evaluation of the automatic color correction module}

Automatic correction was verified in the second step (online) part of Fig. 6. The correct value of the color chart data was measured with a color difference meter (Konica Minolta CS-150) under a D50 light source, referring to the lighting environment of the doctor's office. For evaluation, ten types of color chart images were taken, using two smartphones

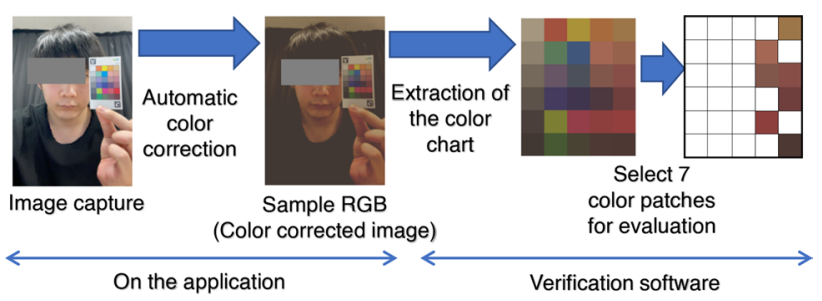

Fig. 7 Procedure for extracting the seven color patches for comparison

(iPhone 8 and iPhone 12), each at five indoor locations.

We used our software written in Python to compare the auto-corrected color patches with the correct values. Figure 7 shows the contents of the software procedure. The software extracted part of the color chart from the corrected image, using AR markers as a guide and obtained data for seven patches. Each of the seven patches was averaged and converted to $L^{*} a^{*} b^{*}$ values using OpenCV's cvtColor. The obtained data were used as test data.

The corrected values and test data were compared using Eq. (1), and the color difference $\Delta \mathrm{E}$ in the $L^{*} a^{*} b^{*}$ space.

$\Delta E=\sqrt{\left(L_{1}^{*}-L_{2}^{*}\right)^{2}+\left(a_{1}^{*}-a_{2}^{*}\right)^{2}+\left(b_{1}^{*}-b_{2}^{*}\right)^{2}}$

where $L_{1}{ }^{*} a_{1}{ }^{*} b_{1}{ }^{*}$ is the reference RGB data, and $L_{2}{ }^{*} a_{2}{ }^{*} b_{2}{ }^{*}$ is the test data value.

Table 3 summarizes the experimental results of automatic color correction. The data before the correction showed a significant color difference, whereas the data after correction showed that $\Delta E$ was within 8 . When $\Delta \mathrm{E}$ was less than 5 , the data could be considered identical, but two patches had a $\Delta E$ greater than 5. These patches were darker in color.

Table 4 summarizes the data after automatic color correction, including the maximum error value, to examine its practical use. Even in the data where the average value of $\Delta \mathrm{E}$ was below 5 , some cases with a maximum value of $\Delta \mathrm{E}$ exceeding five were observed. 
Table 3 Comparison of color difference before and after color correction

\begin{tabular}{lll}
\hline Patch number & Average $\Delta E$ & \\
\cline { 2 - 3 } & Original & Correction \\
\hline E-a Tang & 44.5 & 4.9 \\
D-d Tang & 35.4 & 3.2 \\
E-d Tang & 23.7 & 5.5 \\
E-c Tang & 30.9 & 4.3 \\
E-e Tang & 29.2 & 3.0 \\
E-e Tang & 24.8 & 7.5 \\
D-b Skin & 42.8 & 3.8 \\
\hline
\end{tabular}

Table 4 Correction results including the maximum error value of $\Delta E$

\begin{tabular}{lllrl}
\hline Patch number & Average & \multicolumn{3}{l}{$\Delta E$} \\
\cline { 3 - 5 } & & Min & Max & SD \\
\hline E-a Tang & 4.9 & 1.7 & 13.2 & 3.3 \\
D-d Tang & 3.2 & 1.0 & 7.0 & 2.0 \\
E-d Tang & 5.5 & 1.0 & 9.5 & 2.7 \\
E-c Tang & 4.3 & 1.4 & 10.2 & 2.6 \\
E-e Tang & 3.0 & 1.0 & 5.9 & 1.5 \\
E-e Tang & 7.5 & 5.1 & 11.5 & 1.9 \\
D-b Skin & 3.8 & 2.4 & 9.4 & 2.0 \\
\hline
\end{tabular}

\subsection{Accuracy evaluation of the pulse wave measurement module}

Several methods for pulse wave acquisition using RGB cameras have been proposed. Green $(\mathrm{G})$ signals have been reported to result in an extremely high AC/DC ratio of the PPG waveform $[15,16]$. Accordingly, an accuracy evaluation was performed by comparing the implemented hemoglobin signal with the $\mathrm{G}$ signal.

\subsubsection{Pulse wave measurement method}

The method we implemented is based on Tsumura et al.'s method [13, 14], which converts RGB signals into three signals (hemoglobin, melanin, and shading) and utilizes the changes in the hemoglobin signal to acquire a stable pulse wave without the influence of luminance changes. In this method, the shading components are separated, resulting in higher accuracy than methods that are affected by motion and light sources.

\subsubsection{Acquisition of RGB signals}

The pulse wave signal is detected by capturing the change in the weak signal from the living body. In the iOS normal video recording mode, weak signals are challenging to capture because of the automatic correction and compression of the video. The implemented software directly controls the camera system to acquire uncompressed RGB signals.

\subsubsection{Acquisition of vital information from the RGB signals}

Figure 8 shows a method for obtaining vital information from a video image. First, the region of interest (ROI) that is applicable for pulse wave detection is selected from the total pixels and separated into temporal images. Then, each image is separated into hemoglobin, melanin, and shadow component images using independent component analysis. The average value of the pixel values was calculated from the separated hemoglobin component pixels. Because the change in hemoglobin quantity is correlated with the pulse wave, the pulse wave can be calculated from the time variation of the mean hemoglobin value. To obtain a pulse wave signal with high accuracy, a band-pass filter was used. A pulse wave peak was detected from the obtained signal. The pulse rate was calculated from the average value of the $R-R$ interval.

\subsubsection{Separation of hemoglobin components}

An overview of the hemoglobin component separation is shown in Fig. 9. Independent component analysis (ICA) is a method for finding hidden factors and components in multidimensional data. The difference between ICA and other methods is that the components to be searched are statistically independent and non-Gaussian [17]. Assuming that $t$ is the time and the RGB signal (observed value) is generated

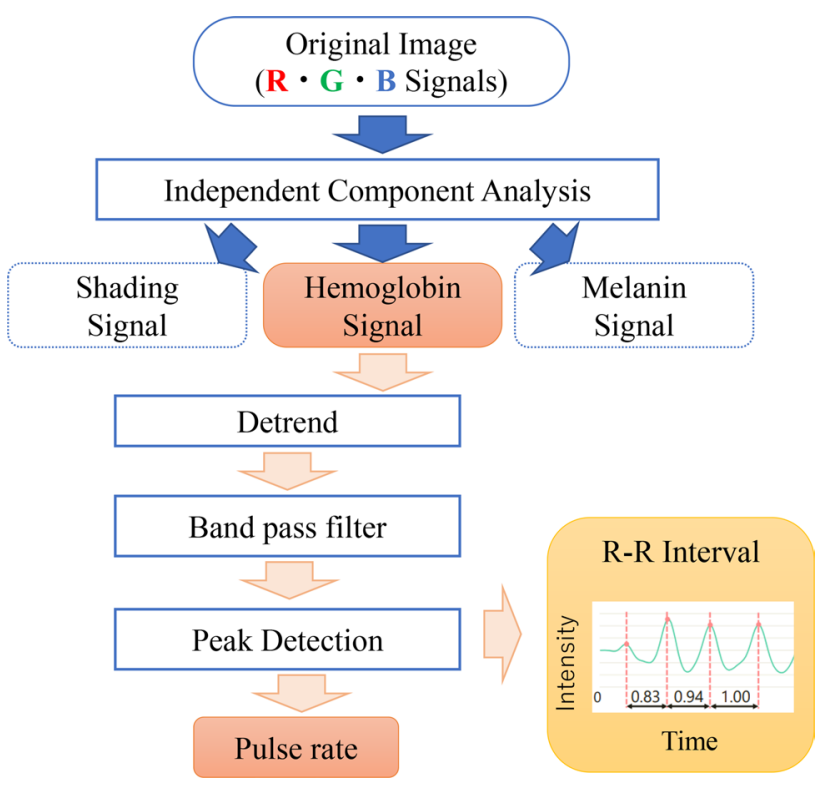

Fig. 8 Overview of Signal Detection Algorithm 


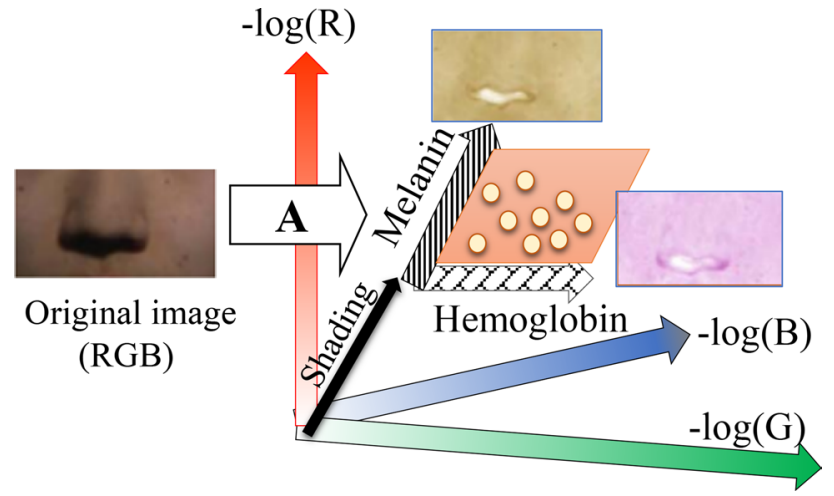

Fig. 9 Overview of the separation of hemoglobin components using ICA

by a linear mixture of hemoglobin, melanin, and shade components, which are independent of each other, the signal can be expressed as in Eq. (2).

$\left(\begin{array}{l}R(t) \\ G(t) \\ B(t)\end{array}\right)=\mathbf{A}\left(\begin{array}{c}H(t) \\ M(t) \\ S(t)\end{array}\right)$

where $R(t), G(t)$, and $B(t)$ are the RGB signals, and $H(t)$, $M(t)$, and $S(t)$ are the hemoglobin, melanin, and shade signals, respectively. Once transformation matrix A can be calculated, the signals can be separated. For the RGB signals, a logarithm was used. Since the three separated components are orthonormal, the hemoglobin component is separated from the shadow component, and it is robust to illumination variations. In addition, the melanin component does not change in the range of measurement time; therefore, the variation in hemoglobin can be extracted.

\subsubsection{Verification of the accuracy of the pulse waveform data}

The iPhone 8 was used as a video recording device for accuracy verification. Because the developed application can obtain both RGB signals and hemoglobin signals calculated from the RGB signals in the CSV format, we used these two signals as the dataset for comparison. We used two types of shooting environments: a stationary state under LED illumination without flicker, and the other was a moving state under 3-wavelength fluorescent light, a typical shooting environment. The video was taken without a chin rest and with a smartphone fixed in place.

The accuracy verification results are shown in Fig. 10. The plotted signal was obtained before denoising. The hemoglobin intensity was plotted as the reciprocal of the $G$ signal because the absorption of the $G$ signal increases
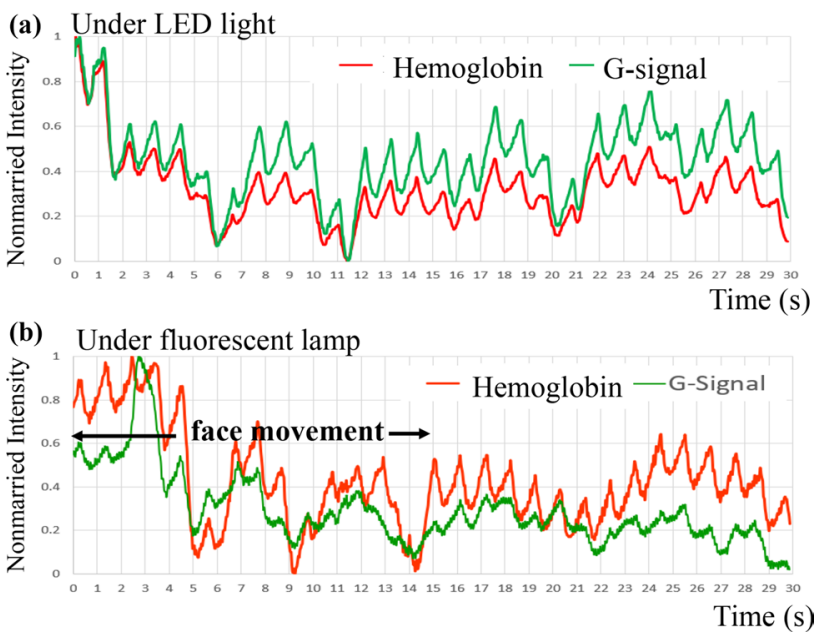

Fig. 10 Verification results of pulse wave extraction accuracy. a Under LED light b under fluorescent lamp

when the hemoglobin content is high. Both signals were normalized.

Figure $10 \mathrm{~b}$ is the signal obtained from a movie taken in a fluorescent light environment. During the first $15 \mathrm{~s}$ of the measurement, the face was slowly moved up and down, left and right. In the next $15 \mathrm{~s}$, the face was kept still.

Under LED illumination and stationary conditions, the hemoglobin and $\mathrm{G}$ signals were almost the same. However, under fluorescent illumination and motion, the hemoglobin waveform was more robust to motion than the $G$ signal, as can be observed from the waveform.

\subsection{Verifying the accuracy of pulse rate detection}

The experiments were conducted in conjunction with a clinical trial to verify the accuracy of pulse rate detection at Kanazawa University to investigate the effects of needle treatment. [Medical Ethics Committee of Kanazawa University (2018-154)].

The experiment was conducted in the Kampo consultation room of Shinseikai Toyama Hospital, and the subjects were 39 nurses who gave their consent. Measurements were taken before and after needle treatment. We conducted two types of lighting, one with a fluorescent lamp and the other with an LED lamp, and the measurement time for each was $30 \mathrm{~s}$. The setup of the system was the same as that used in the preliminary experiment conducted at the Chiba University campus (Fig. 11). A VILTROX L116T RA CRI 95 + Super Slim LED Light Panel was used as the LED light, and the color temperature was set to $5600 \mathrm{~K}$. The fluorescent light was a 3-wavelength fluorescent light for room lighting in the examination room. The frame rate of the iPhone XR was set to $60 \mathrm{fps}$. 


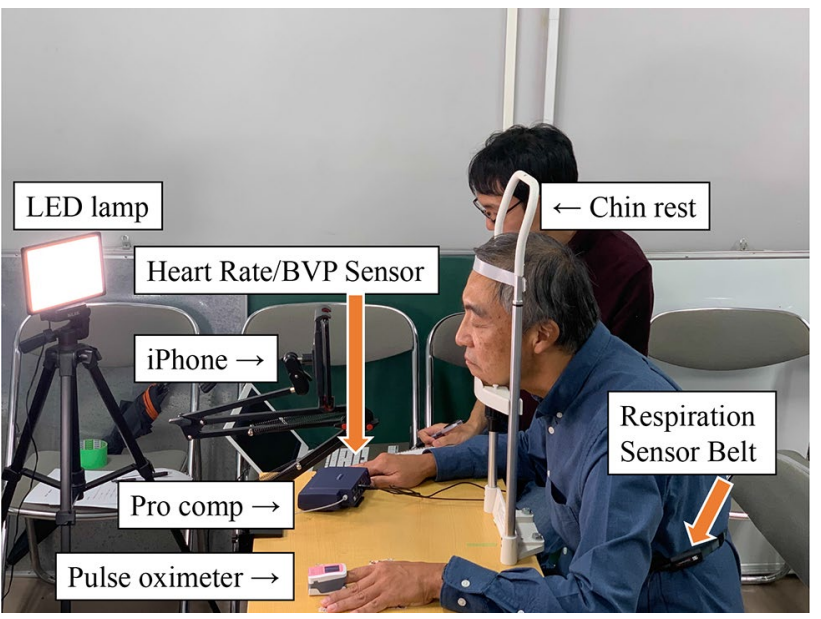

Fig. 11 Setting for preliminary experiments

The correct values were obtained using the ProComp system (Thought Technology, Canada). ProComp was controlled by a laptop computer running Windows 10 with ProComp-specific software to capture the data. The pulse rate measurement device connected to ProComp was a fingertip photoplethysmograph (PPG) sensor (Thought Technology Heart Rate/BVP Sensor) using infrared rays, and data sampling was performed at $2048 \mathrm{~Hz}$. The pulse rate was calculated from the pulse wave signal obtained, using a program written in Python.

We also used a two-wavelength blood absorption pulse oximeter (PO) (Pulsfit BO-650 NISSEI) for pulse rate measurements as a comparison target. As the PO cannot acquire continuous pulse wave signals, the values 15 and $30 \mathrm{~s}$ after the start of the experiment were read visually, and the average value was used.

The heart rate measurement results under fluorescent light before and after treatment are shown in Fig. 12. The results of three subjects showing evident abnormal values were removed from the experimental results, and the data of 36 subjects were used. In Fig. 12, the vertical axis of the heart rate is shown for the 36 subjects. The experimental data before needle treatment showed that the accuracy was within the range where the differences between subjects could be discerned. The post-treatment data showed a decrease in the heart rate compared with the pre-treatment data. The accuracy of the data showed good consistency, except for subject 6 . All four types of experimental data are presented in Fig. 13. The graphs show the iPhone and PO data plotted in a scatter plot against the heart rate obtained from PPG. The upper and lower auxiliary lines indicate an error rate (Er) of 10\%, which was calculated using Eq. (3).

$\mathrm{Er}=\frac{\text { measured_data }- \text { PPG data }}{\text { PPG data }} \times 100$

where measured data were calculated from iPhone and PPO. (a) measured pulse rate data under

fluorescent lamp ( Before needle treatment)

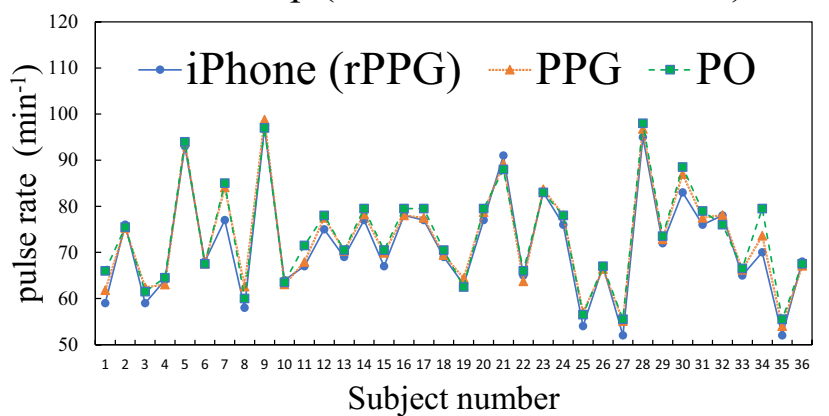

(b) measured pulse rate data under

fluorescent lamp (After needle treatment)

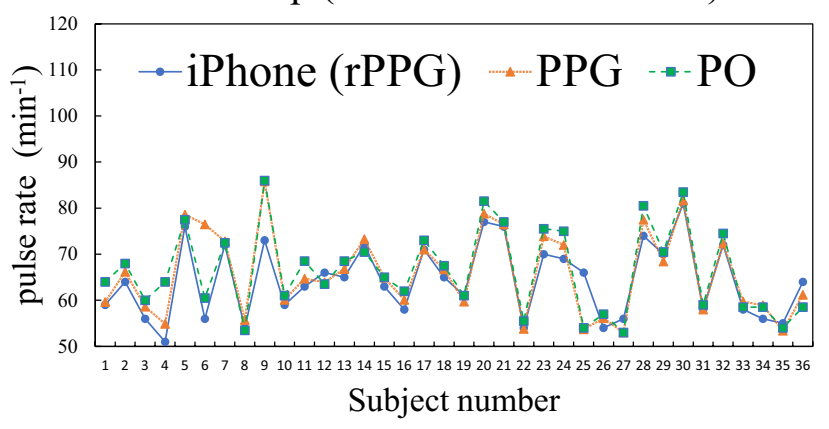

Fig. 12 Accuracy verification results for the pulse rate

The case data of fluorescent and LED lamps before needle treatment showed that the Er of the iPhone was almost within $10 \%$, and the correlation coefficient was 0.99 . This indicates that the accuracy was almost equal to that of the PO.

Figure 13 shows the experimental data after the needle treatment, where the pulse rate was relatively low. The accuracy of the data under fluorescent light immediately after needle treatment was lower than that under the other three conditions.

The error rates and correlation coefficients were almost the same for iPhone and PO.

Next, we will discuss the comparison results between the $G$ signal and the hemoglobin signal obtained using ICA. Since the system used in this experiment records each RGB signal separately, which is the original data during video recording, the recorded $G$ signal was used for analysis. The process for counting pulse rate from the $G$ signal, i.e., smoothing, bandpass filtering, pulse wave peak detection, and pulse rate counting, used the same algorithm as the process for counting pulse rate from hemoglobin signals. However, the processing of the $G$ signal was analyzed using Python on a PC. The processing of the hemoglobin signal, on the other hand, was conducted in an iOS, with a different module for the Fourier transform. 


\section{Before needle treatment}

(Under florescent lamp)

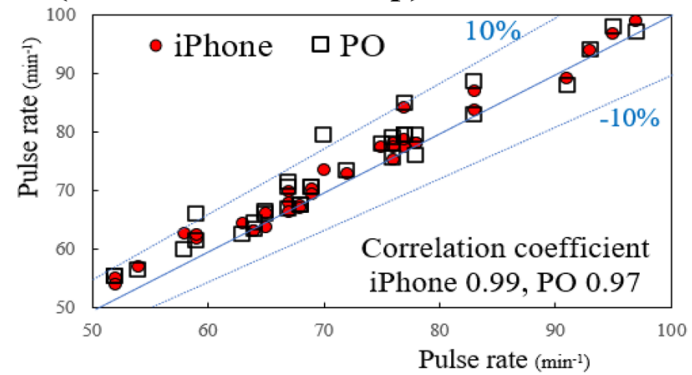

Before needle treatment (Under LED light)

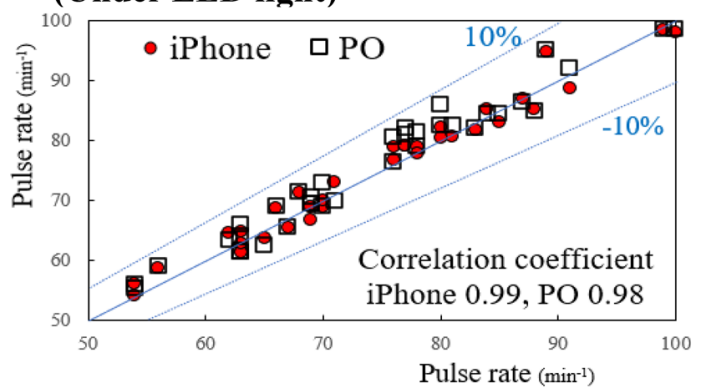

After needle treatment

(Under florescent lamp)

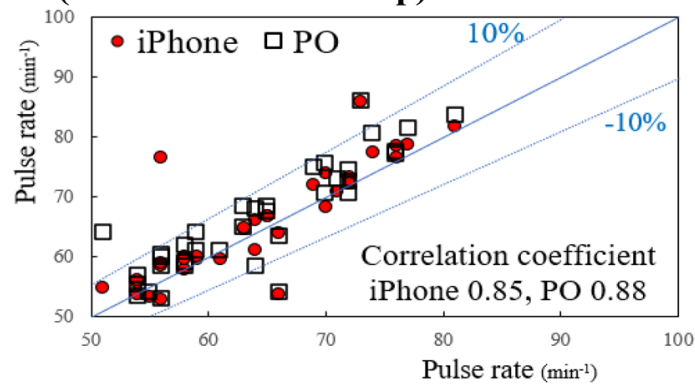

After needle treatment

(Under LED light)

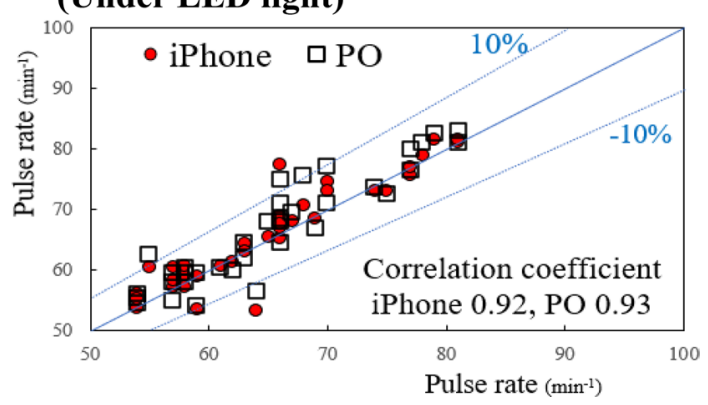

Fig. 13 Accuracy verification results for the pulse rate

The true value is PPG, and the correlation of pulse rate counts was performed under the same four conditions as in the previous chapter. The experiment results are shown in Table 5. The correlation factor was almost the same for both the $\mathrm{G}$ and the hemoglobin signals. In this case, since we use
Table 5 Correlation of heart rate obtained from $G$ signals and hemoglobin signals to the true value

\begin{tabular}{llllll}
\hline & \multicolumn{2}{l}{ Fluorescent lamp } & & \multicolumn{2}{l}{ LED light } \\
\cline { 2 - 3 } \cline { 5 - 6 } & $G$ signal & $\begin{array}{l}\text { Hemo- } \\
\text { globin } \\
\text { signal }\end{array}$ & & $G$ signal & $\begin{array}{l}\text { Hemo- } \\
\text { globin } \\
\text { signal }\end{array}$ \\
\hline Before treatment & 1.00 & 0.99 & 0.99 & 0.99 \\
After treatment & 0.90 & 0.85 & & 0.99 & 0.92 \\
\hline
\end{tabular}

a chin rest, we can assume that there is no effect of shading caused by movement.

\subsection{Comprehensive evaluation of iOS-based telemedicine tool}

In this section, we present the results of Survey results for application improvement on doctors to evaluate the tool. The evaluation was conducted in August 2021 for doctors, mainly in Hiroshima and Shizuoka prefectures. The doctors used this telemedicine tool for multiple days and submitted questionnaires after completion. Submissions were accepted either anonymously or unnamed. The evaluation items were operability, rated in three levels (easy to understand, normal, and difficult to understand). In addition, a free entry column was provided.

The results are shown in Table 6. A total of three questions were asked and responses were rated at three different sensitivity levels. More than $80 \%$ of the respondents answered normal or above.

The following summarizes the comments made in the free comments section. Regarding face and tongue photo shooting, there was a comment that "it was difficult to shoot while holding the color chart".

Regarding the operation of the application, several comments mentioned that "it was difficult to install the application". However, there were no negative comments about the telemedicine tool itself, and the participants agreed that the accumulation of such data is important.

Table 6 Survey results for application improvement

\begin{tabular}{llll}
\hline & $\begin{array}{l}\text { Easy to } \\
\text { understand } \\
(\%)\end{array}$ & Normal (\%) & $\begin{array}{l}\text { Difficult to } \\
\text { understand } \\
(\%)\end{array}$ \\
\hline $\begin{array}{l}\text { Face and tongue photo } \\
\text { shooting }\end{array}$ & 55 & 30 & 15 \\
$\begin{array}{l}n=33) \\
\begin{array}{l}\text { Vital measurement } \\
(n=34)\end{array}\end{array}$ & 62 & 38 & 0 \\
$\begin{array}{l}\text { Operation of the application } \\
(n=34)\end{array}$ & 26 & 62 & 12 \\
\hline
\end{tabular}




\section{Discussion}

Telemedicine increased after the COVID-19 pandemic, where "forward triage," the sorting of patients before they arrive in the emergency department (ED), is the most important and emerging medical need. For example, respiratory symptoms that may be early signs of COVID-19 are among the most evaluated with this approach. Telemedicine may be a virtually perfect solution. Telemedicine with high quality can allow physicians and patients to communicate $24 \mathrm{~h}$ a day, using smartphones or webcam-enabled computers. At the same time, physicians can observe the color of the face, lips, or tongues.

It is reported that telephone consultations typically convey less information than video consultations and are reimbursed at a fraction of a comparative video telehealth consultation [18].

Natural face color is one of the most important physical examination points.

Our previous studies demonstrated that tongue color reflects the diagnosis in Kampo medicine [19], and evaluation of Kampo Disease State is possible with images of the face and tongue [20,21]. Facial color diagnosis is also an important diagnostic method in Kampo medicine and traditional Chinese medicine (TCM). Another study showed that face color diagnosis can properly identify patients with hepatitis into three groups (healthy, severe hepatitis with jaundice, and severe hepatitis without jaundice) with accuracy higher than $73 \%$ [22]. We believe that the usefulness of the color chart-based telemedicine tool will increase, and we will continue to improve it further.

The automatic color correction of the color chart was evaluated by determining the difference between Ref. RGB and the corrected samples. Sample images with a significant error $\Delta E$ were considered to have inaccurate interpolation because of the narrow of $\Delta E$ between the white and black patches of that image. To examine the relationship between the magnitude of the error and the color difference between the white patch (A-a) and the black patch (A-f), all ten conditions, which are the test data, are described in Fig. 14. The horizontal axis shows the color difference $\Delta \mathrm{E}$ between the white patch (A-a) and the black patch (A-f), and the vertical axis shows the number of patches with the correct error difference $\Delta E$ greater than 6 . Because we evaluated seven different patches for one test data sample, the maximum number of patches on the vertical axis was seven. The smaller the $\Delta E$ of the black and white patches, the larger the error. The difference between the models used for photography was that the iPhone 8 had a smaller $\Delta E$ than the iPhone 12 , but the number of errors was not significantly different. In the future, we would like to conduct additional experiments to reduce the $\Delta E$.

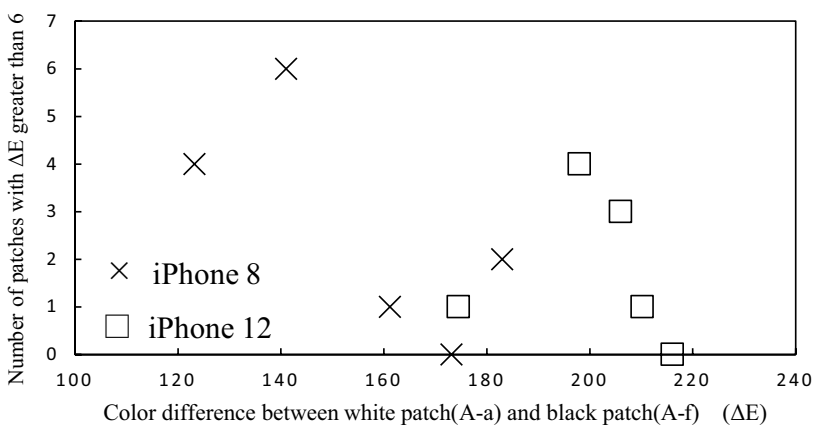

Fig. 14 Relationship between the number of errors and the color difference between white and black patches

It is necessary to calibrate the monitor for accurate color reproduction. By using a calibration tool for commercially available monitors, accurate color reproduction can be achieved.

Automatic color correction helps to compare images between patients, even if the monitor is not calibrated. In this system, doctors use color charts, so they can recognize the differences in colors. We believe that this system will work effectively as long as the physician understands the characteristics of the proposed method. In a future study, we will address the simplification of the calibration of the monitor. We are currently developing a simple correction method using a mirror. We examined the possibility of performing color correction by simultaneously photographing the color chart and the doctor's monitor.

The accuracy of the pulse wave signal obtained from the captured images, the comparison of the hemoglobin signal, and the $G$ signal showed that the hemoglobin signal was robust against motion.

As a result of verifying the accuracy of the implemented system with 36 subjects, the correlation coefficient with the correct answer values was equivalent to that of the PO, confirming the effectiveness of this system. The accuracy of the data under fluorescent light after acupuncture was poor compared with the other conditions. This result may have been due to the effect of needle treatment. Needle treatment was performed for approximately $30 \mathrm{~min}$. After the needle treatment, the patient was fitted with a sensing device for approximately $2 \mathrm{~min}$, and the tongue and face were photographed under fluorescent light. Next, video recording for vitals was performed under fluorescent light for $30 \mathrm{~s}$, followed by measurements under LED light, for tongue and face. The pulse wave was obtained from the changes in the hemoglobin signal. The decrease in the detection accuracy may be due to the decrease in blood pressure resulting from the drowsiness owing to the needle treatment, which reduces the range of change in the hemoglobin signal. The measurement time under LED illumination after needle treatment was a few minutes after the measurement under fluorescent 
illumination. The error may have decreased as blood pressure returned to normal over time.

In this verification, there were some data where the true value, PPG, was not certain. That is the case where the iPhone and PO data were the same but only the PPG data was different. To detect biological signals, methods for isolating weak signals are essential. Since many signal processing methods have been proposed [23], in the future, we plan to utilize signal processing methods to find a way to obtain a more reliable pulse wave.

\section{Conclusion}

We implemented a non-contact telemedicine tool for infection prevention in iOS and conducted an accuracy verification experiment. As a result, the doctors who participated in this study evaluated the tool as being at a level where they would like to try it out in the medical field. Because the tool can be used with commercially available iPhones, the range of use will expand. In the future, we plan to test and improve the tool using it in conjunction with clinical trials.

Acknowledgements We would like to thank Dr. Miki Harada of Shinseikai Toyama Hospital and Ms. Mako Iwahashi of Hiroshima University Hospital for their cooperation in the evaluation of this study. The color charts were created by the DIC Corporation and DIC Graphics Corporation. We express our gratitude to them.

\section{References}

1. BCC Research (2018) Global telehealth market. https://www. bccresearch.com/partners/verified-market-research/global-teleh ealth-market-vmr235a.html. Accessed 20 Jan 2022

2. Greenhalgh T, Wherton J, Shaw S, Morrison C (2020) Video consultations for COVID-19. BMJ 368:m998. https://doi.org/10.1136/ bmj.m998

3. Hollander JE, Carr BG (2020) Virtually perfect? Telemedicine for COVID-19. N Engl J Med 382(18):1679-1681

4. Baum A, Kaboli PJ, Schwartz MD (2021) Reduced in-person and increased telehealth outpatient visits during the COVID-19 pandemic. Ann Internal Med 174(1):129-131

5. Majumder S, Deen MJ (2019) Smartphone sensors for health monitoring and diagnosis. Sensors 19(9):2164

6. Singh AK, Kasle DA, Jiang R, Sukys J, Savoca EL, Lerner ZM, Kohli N (2021) A review of telemedicine applications in otorhinolaryngology: considerations during the coronavirus disease of 2019 pandemic. Laryngoscope 131(4):744-759

7. Meng X, Dai Z, Hang C, Wang Y (2020) Smartphone-enabled wireless otoscope-assisted online telemedicine during the
COVID-19 outbreak. Am J Otolaryngol. https://doi.org/10.1016/j. amjoto.2020.102476

8. Nishibori M (2000) Problems and solution in medical color imaging. In: Proceedings of the second international symposium on multi-spectral imaging and high accurate color reproduction, pp 9-17

9. Morishita M, Takahashi O, Yoshii S, Hayashi M, Kibune R, Nakamura T, Awano S (2021) Effect of COVID-19 on dental telemedicine in Japan. J Dental Sci 17(1):42-48

10. Ministry of Health, Labour and Welfare (2018) Guidelines for the appropriate implementation of online medical treatment. https:// www.mhlw.go.jp/file/05-Shingikai-10801000-Iseikyoku-Soumu $\mathrm{ka} / 0000201789$.pdf. Accessed 20 Jan 2022 (in Japanese)

11. Ogawa K (2020) Kampo medicine: the first step to Ching Kyobo Theory. Zen-nihonbyoin shuppan kai (ISBN 9784865192759, in Japanese)

12. Takahashi M, Takahashi R, Morihara Y, Kin I, Ogawa-Ochiai K, Tsumura N (2020) Development of a camera-based remote diagnostic system focused on color reproduction using color charts. Artif Life Robot 25(3):370-376

13. Fukunishi M, Kurita K, Yamamoto S, Tsumura N (2017) Non-contact video-based estimation of heart rate variability spectrogram from hemoglobin composition. Artif Life Robot 22(4):457-463

14. Fukunishi M, Mcduff D, Tsumura N (2018) Improvements in remote video-based estimation of heart rate variability using the Welch FFT method. Artif Life Robot 23(1):15-22

15. Verkruysse W, Svaasand LO, Nelson JS (2008) Remote plethysmographic imaging using ambient light. Opt Express 16(26):21434-21445

16. Maeda Y, Sekine M, Tamura T (2011) The advantages of wearable green reflected photoplethysmography. J Med Syst 35(5):829-834

17. Hyvärinen A, Oja E (2000) Independent component analysis: algorithms and applications. Neural Netw 13(4-5):411-430

18. Contreras CM, Metzger GA, Beane JD, Dedhia PH, Ejaz A, Pawlik TM (2020) Telemedicine: patient-provider clinical engagement during the COVID-19 pandemic and beyond. J Gastrointest Surg 24(7):1692-1697

19. Yamamoto S, Ishikawa Y, Nakaguchi T, Ogawa-Ochiai K, Tsumura N, Kasahara Y, Namiki T, Miyake Y (2012) Temporal changes in tongue color as criterion for tongue diagnosis in Kampo medicine. Complement Med Res 19(2):80-85

20. Matsushita F, Kiyomitsu K, Ogawa K, Tsumura N (2019) Evaluation of Kampo disease state using facial images. Artif Life Robot 24(1):44-51

21. Koike R, Ogawa-Ochiai K, Shirai A, Hayashi K, Arimitsu J, Li $\mathrm{H}$, Tsumura N (2021) Creating a diagnostic assistance system for diseases in Kampo medicine. Appl Sci 11(21):9716. https://doi. org/10.3390/app11219716

22. Liu M, Guo Z (2008) Hepatitis diagnosis using facial color image. In: International conference on medical biometrics, pp 160-167

23. Rouast PV, Adam MT, Chiong R, Cornforth D, Lux E (2018) Remote heart rate measurement using low-cost RGB face video: a technical literature review. Front Comput Sci 12(5):858-872

Publisher's Note Springer Nature remains neutral with regard to jurisdictional claims in published maps and institutional affiliations. 